\title{
The bivariate complete fourth power exponential distribution
}

\author{
Dalal Adnan Maturi \\ Department of Mathematics, Faculty of Science, King Abdulaziz University, Jeddah, Saudi Arabia \\ E-mail: maturi_dalal@yahoo.com
}

\begin{abstract}
Amira and Mazloum [1] studied the Complete Fourth Power Exponential (CFPE) distribution and its geometric and statistical properties against the normal distribution. Maturi \& Elsayigh [2] investigated the relationship between variate values and ranks in samples from the CFPE distribution. In this paper a bivariate version of the CFPE distribution (BCFPE) is introduced and some of its properties are explored.
\end{abstract}

Keywords: Bivariate Distribution, Complete Fourth Power Exponential Distribution, Normal Distribution.

\section{Introduction}

Amira and Mazloum [1] introduced the Complete Fourth Power Exponential (CFPE) distribution and its geometric and statistical properties against the normal distribution. Ibrahim [3] proposed estimators for the two parameters of the CFPE distribution using the maximum likelihood method and the moments method. Baeshen [4], in her master dissertation, studied the misclassification of a random sample drawn from the CFPE distribution as to be belong to the normal distribution rather from the CFPE distribution. Maturi and Elsayigh [2] studied the relationship between the variate values in samples from the CFPE distribution and the corresponding ranks, in which they found that the correlation is relatively high, equal to 0.728. Recently, a closed simple formula for the characteristic function of this distribution is given by Pogány [5].

The outline of this paper is as follows. In section 2 we briefly introduce the CFPE distribution as presented in Amira and Mazloum [1] and Maturi and Elsayigh [2]. The bivariate version of the CFPE distribution (BCFPE) is introduced in section 3 and some of its properties are explored. Finally, some concluding remarks are included in section 4 .

\section{The complete fourth power exponential (CFPE) distribution}

In this section we briefly introduce the CFPE distribution as presented in Amira and Mazloum [1] and Maturi and Elsayigh [2]. Let $X$ be a random variable from the CFPE distribution with the probability density function given by,

$$
f(x ; \alpha, \beta)=\frac{2}{\beta \Gamma\left(\frac{1}{4}\right)} \exp \left\{-(x-\alpha)^{4} / \beta^{4}\right\}
$$

where $-\infty<x<\infty,-\infty<\alpha<\infty, \beta>0$ and $\alpha$ and $\beta$ are the location and the scale parameters, respectively. The characteristic function of this distribution is given by

$$
\phi_{X}(t)=\sum_{k=0}^{\infty} \sum_{j=0}^{\left[\frac{k}{2}\right]} C_{2 j}^{k} \alpha^{(k-2 j)} \beta^{2 j} \frac{\Gamma\left(\frac{2 j+1}{4}\right)}{\Gamma\left(\frac{1}{4}\right)} \frac{(i t)^{k}}{k !}
$$


where $[k / 2]$ is the greatest integer number less than $k / 2$. Thus, the mean and the variance of the CFPE distribution are

$$
\mu=E(X)=\alpha \quad \text { and } \quad \sigma^{2}=\beta^{2} \frac{\sqrt{2} \pi}{\left[\Gamma\left(\frac{1}{4}\right)\right]^{2}}
$$

A closed simple formula of the above characteristic function is given by Pogány [5].

\section{The bivariate complete fourth power exponential distribution}

In this section the bivariate CFPE distribution is introduced and some of its properties are explored. The probability density function of the random variable $(X, Y)$ from the bivariate CFPE distribution is given as

$$
\begin{array}{r}
f\left(x, y ; \alpha_{x}, \alpha_{y}, \beta_{x}, \beta_{y} ; \rho\right)=\frac{2}{\beta_{x} \beta_{y} \pi \sqrt{\pi} \sqrt{1-\rho^{2}}} \exp \left\{\frac { - 1 } { ( 1 - \rho ^ { 2 } ) ^ { 2 } } \left[\left(\frac{x-\alpha_{x}}{\beta_{x}}\right)^{2}+\left(\frac{y-\alpha_{y}}{\beta_{y}}\right)^{2}\right.\right. \\
\left.\left.-2 \rho\left(\frac{x-\alpha_{x}}{\beta_{x}}\right)\left(\frac{y-\alpha_{y}}{\beta_{y}}\right)\right]^{2}\right\}
\end{array}
$$

where $-\infty<x<\infty,-\infty<y<\infty,-\infty<\alpha_{x}<\infty,-\infty<\alpha_{y}<\infty, \beta_{x}>0, \beta_{y}>0$. The correlation coefficient $-1 \leq \rho \leq 1$ describes the strength of the relationship between the two random variables $X$ and $Y$. The mean vector $\mathbf{M}$ and the covariance matrix $\boldsymbol{\Sigma}$ of the bivariate CFPE distribution are given by

$$
\begin{aligned}
& \mathbf{M}=E\left[\begin{array}{l}
X \\
Y
\end{array}\right]=\left[\begin{array}{l}
\alpha_{x} \\
\alpha_{x}
\end{array}\right] \\
& \boldsymbol{\Sigma}=\operatorname{Var}\left[\begin{array}{l}
X \\
Y
\end{array}\right]=\frac{1}{2 \sqrt{\pi}}\left[\begin{array}{cc}
\beta_{x}^{2} & \rho \beta_{x} \beta_{y} \\
\rho \beta_{x} \beta_{y} & \beta_{y}^{2}
\end{array}\right]
\end{aligned}
$$

The density function $f\left(x, y ; \alpha_{x}, \alpha_{y}, \beta_{x}, \beta_{y} ; \rho\right)$ in (2) satisfies the probability distribution function properties [6], namely

$$
\int_{x} \int_{y} f\left(x, y ; \alpha_{x}, \alpha_{y}, \beta_{x}, \beta_{y} ; \rho\right) d x d y=1
$$

and

$$
f\left(x, y ; \alpha_{x}, \alpha_{y}, \beta_{x}, \beta_{y} ; \rho\right) \geq 0 \quad \text { for } \quad-\infty<x<\infty,-\infty<y<\infty .
$$

As usual the marginal distribution in (1) (i.e. for $X$ ) can be obtained from (2) by integrating over the $Y$ range. Similarly the marginal distribution of $Y$ can be obtained by integrating over the $X$ range.

Fig.1 shows the standard (univariate) normal distribution $N(0,1)$ with three different (univariate) CFPE distributions at $\sigma=1,0.5,2$ and $\alpha=0$. Clearly, the CFPE distributions have thinner tails and the majority of the values clustered around the mean compared with the standard normal distribution. Fig.2 represents contours of the bivariate standard normal density functions (the diagrams on the left) and the bivariate complete fourth power exponential (BCFPE) density functions (the diagrams on the right, where $\alpha_{x}=\alpha_{y}=0$ ) at different values of the correlation coefficient $\rho$, i.e. $\rho=-0.4$ (negative correlation), $\rho=0$ (no correlation) and $\rho=0.6$ (positive correlation). Again, clearly the values are more centered around the middle in the case of the BCFPE distribution.

\section{Conclusion}

In this paper, we introduced the bivariate version of the Complete Fourth Power Exponential distribution and discussed some of its properties. The BCFPE distribution can be very attractive when the data are seem to be heavily centered around the mean and no outliers are expected. Deriving estimates of the parameters of the BCFPE distribution, e.g. the maximum likelihood estimation and studying the estimators' properties, is left for future research. A similar study to Baeshen [4] can be conducted to investigate the misclassification of random bivariate samples from BCFPE distribution and have been misclassified to be from bivariate normal distribution. The multivariate version of this distribution could be an interesting topic for future research. 


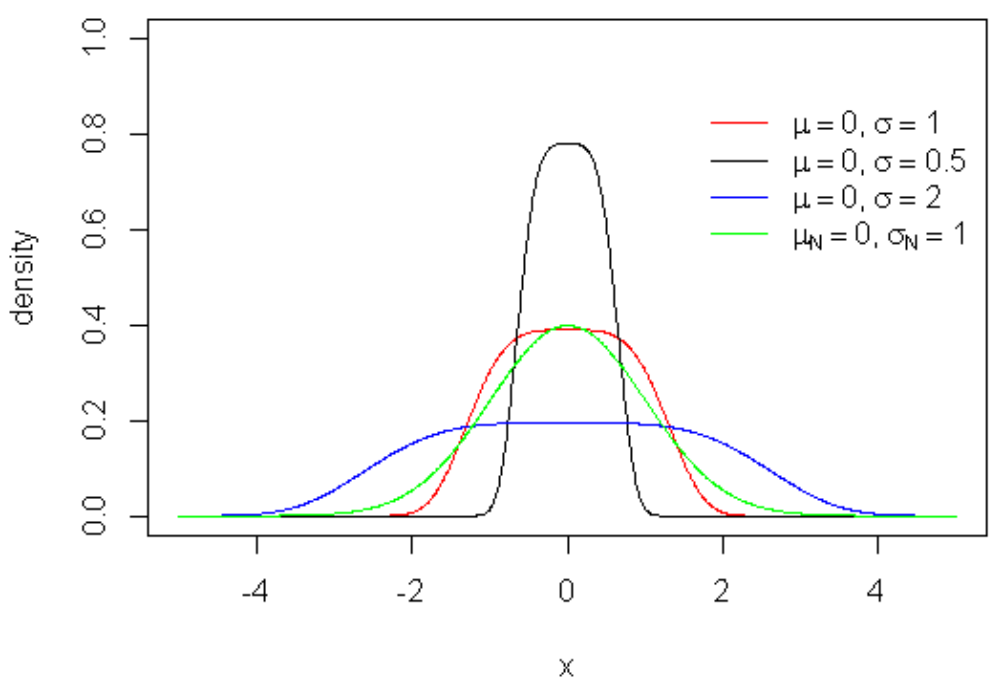

Figure 1: The standard normal distribution $N(0,1)$ with three different CFPE distributions

\section{References}

[1] T. Amira \& R. Mazloum, "Complete Fourth Power Exponential Distribution Geometric and statistical study", Econometric and Business Review, Ain Shams University, (1993).

[2] T.A. Maturi \& A. Elsayigh, "The Correlation between VariateValues and Ranks in Samples from Complete Fourth Power Exponential Distribution", Journal of Mathematics Research, Vol. 1, No. 1, (2009), 14-18.

[3] M. A-R. Ibrahim, "Estimation of location and scale parameters for the complete fourth power exponential distribution", Econometric and Business Review, Ain Shams University, (1995).

[4] M. Baeshen, "Study on the Error in Random Samples Classification between the Normal Distribution and the Complete Fourth Power Exponential", Unpublished MSc thesis, King Abdulaziz University, Saudi Arabia, (2000).

[5] T. K. Pogány, "Closed Expression for Characteristic Function of CEPE Distribution", Journal of Mathematics Research, Vol. 2, No. 2, (2010), 49-50.

[6] J.A. Rice, "Mathematical Statistics and Data Analysis", Wadsworth Publishing Co Inc, (1994). 


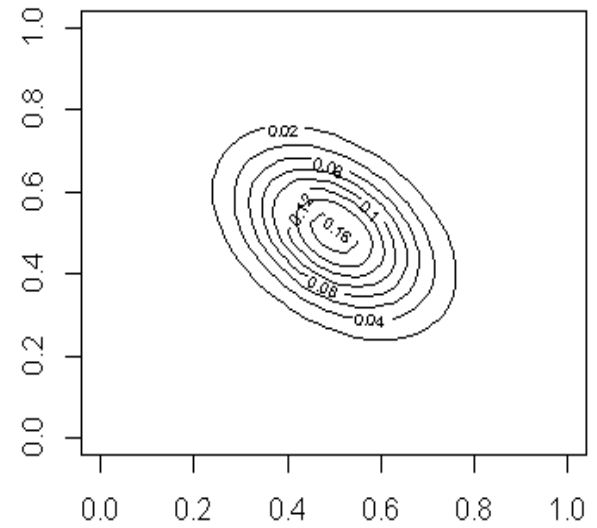

(a) Bivariate normal distribution, $\rho=-0.4$

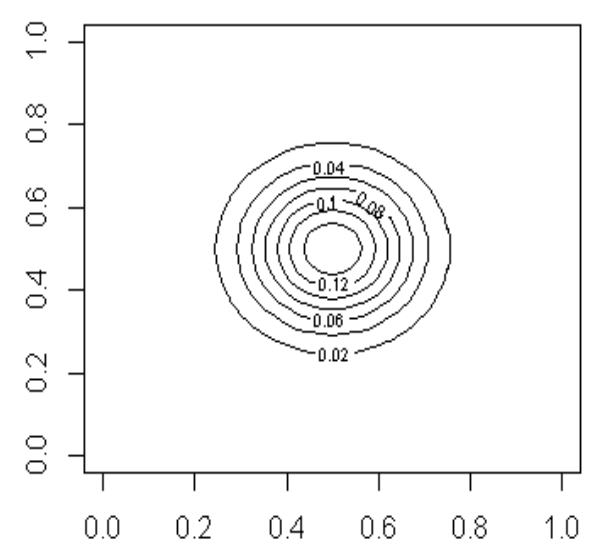

(c) Bivariate normal distribution, $\rho=0$

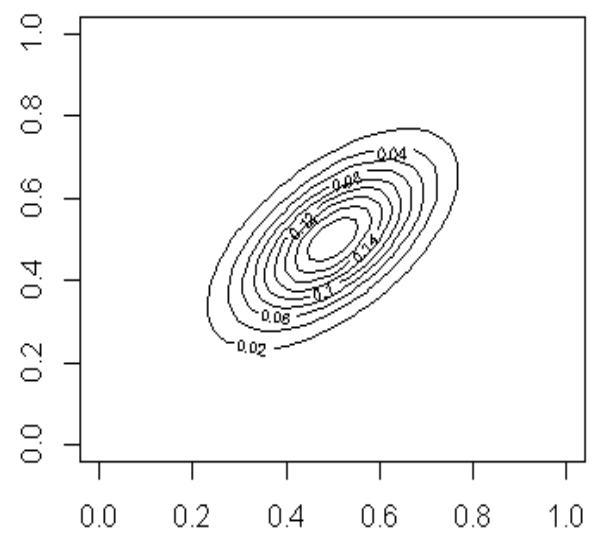

(e) Bivariate normal distribution, $\rho=0.6$

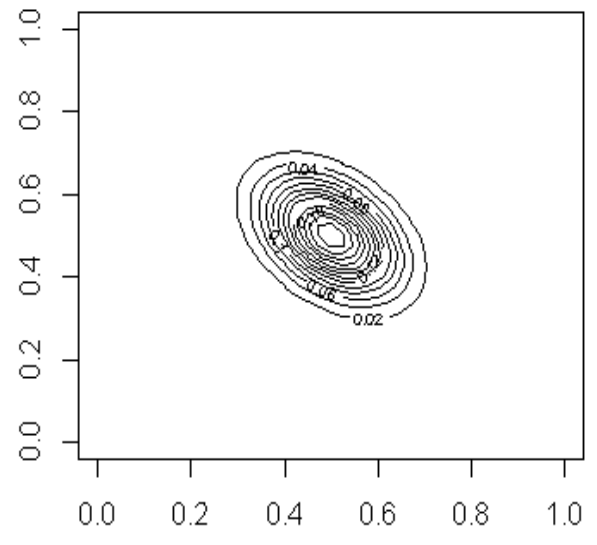

(b) BCFPE distribution, $\rho=-0.4$

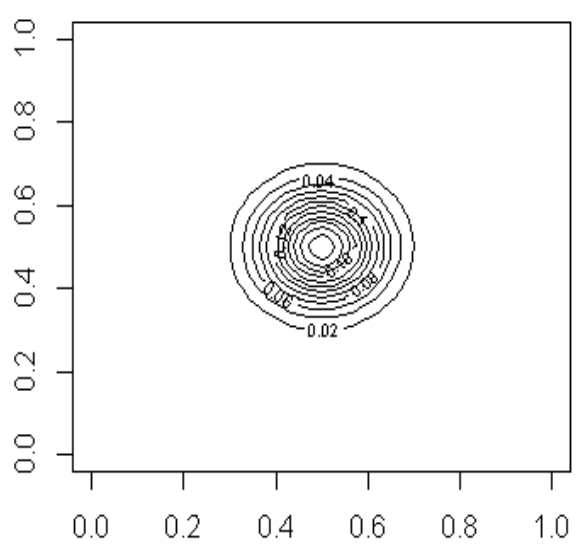

(d) BCFPE distribution, $\rho=0$

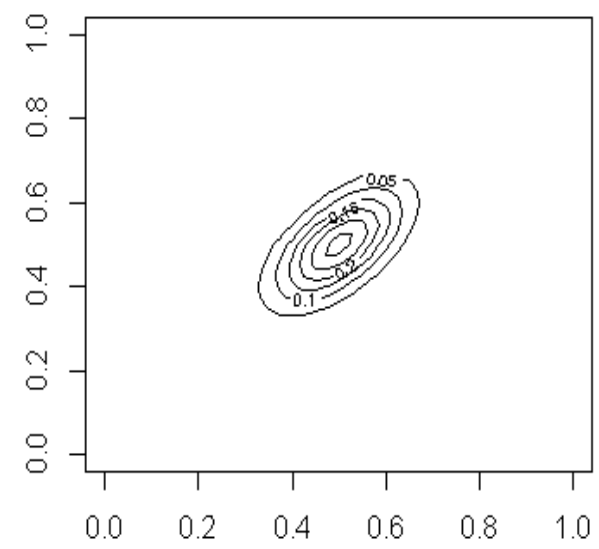

(f) BCFPE distribution, $\rho=0.6$

Figure 2: Bivariate normal distribution (left) and BCFPE distribution (right) at different values of $\rho=-0.4,0,0.6$. 